\title{
Deformation of multilayers and optical surfaces in soft x-ray adaptive optics \\ (Conference Presentation) (Withdrawal Notice)
}

Benjamin Wylie-van Eerd, Huiyu Yuan, Evert Houwman, Oleksandr Antonov, Eric Louis, et al.

Benjamin J. Wylie-van Eerd, Huiyu Yuan, Evert Houwman, Oleksandr Antonov, Eric Louis, Andrey E. Yakshin, Guus J. H. M. Rijnders, Fred Bijkerk, "Deformation of multilayers and optical surfaces in soft x-ray adaptive optics (Conference Presentation) (Withdrawal Notice)," Proc. SPIE 9963, Advances in X-Ray/EUV Optics and Components XI, 99630N (26 October 2017); doi: 10.1117/12.2239018

Event: SPIE Optical Engineering + Applications, 2016, San Diego, California, United States 


\section{Deformation of multilayers and optical surfaces in soft $\mathbf{x}$ - ray adaptive optics(Conference Presentation) (Withdrawal Notice)}

Proc. SPIE 9963, 99630N (2016)

Online Publication Date: 2 November 2016

Withdrawn from Publication: 26 October 2016

Conference Date: 31 August-1 September 2016

Conference Location: San Diego, United States

Conference Title: Advances in X-Ray/EUV Optics and Components XI

Conference Chairs: Ali M. Khounsary, Shunji Goto, Christian Morawe

Benjamin J. Wylie-van Eerd

Univ. Twente (Netherlands)

Publisher's Note: This conference presentation, originally published on 2 November 2016, was withdrawn per author request. 